period and much of the paper on this subject is now out of date.

The seventeen papers devoted to chemical analysis of museum materials cover a number of aspects including outlines of new techniques. The fact that a museum object must go back to its showcase after chemical analysis without any significant damage means that special techniques must be devised. Such methods as X-ray fluorescence and neutron activation come into prominence and there are several examples of these techniques given. The majority of the papers, however, are concerned with particular applications of these techniques. These studies are concerned with attribution or authentication as well as examination of the techniques used in ancient crafts. The range of materials studied and discussed is surprisingly large; analyses of coins, jewellery, bronzes, silverware, ceramics, picture pigments, obsidian, bone and amber are described in one or more of the papers.

When one has looked through this book, one wonders who is likely to buy it for their shelves. It is not written for the specialist since none of the subjects is dealt with in any depth and for the general technical reader it by no means covers the whole field since the papers presented were chosen by the authors and do not fit any particular overall design. It is, however, a most elegant volume. $\quad$ E. T. HALL

\section{Biochemical Technology}

Advances in Biochemical Engineering. Vol. 1. Edited by T. K. Ghose and A. Fiechter. Pp. vii +194 . (Springer Verlag: Berlin and New York, 1971.) 48DM ; \$13.90.

THE series "Advances in Biochemical Engineering" is a very welcome addition to the literature, and I hope it will contribute to the development, to quote the editors, of "the yet to emerge hybrid discipline of biochemical engineering". There are possibly two approaches to be taken in the preparation of a series entitled "Advances in ....", and these depend largely on the nature of the intended audience. The first involves "in depth" critical studies over restricted areas and the second is more generally discursive with the intention of bringing the newer developments to the attention of a wider audience. The first volume in the series falls primarily into the latter category. This by no means detracts from the value of the present volume but would, if continued throughout the series, reduce the potential of its contribution to biochemical engineering. Engineering studies seem to be less amenable to "popular" treatment than either industrial microbiology or even biochemical technology. Unfortunately, the effect of such a presentation of biochemical engineering is to diminish the subject and to identify the potential engineering contribution with gadgetry and empiricism. Biochemical engineering must, if it is to deserve the title, develop into a systematic subject on a simple mathematical basis derived from the laws of conservation and the equations describing transport processes and physical biochemistry. Only in this way will the methodology be established of a rational and progressive discipline.

The first volume in the series covers a wide area including the fluid mechanical properties of fermentation fluids, the recovery of microbial mass from fermentation broths by flocculation and sedimentation, the kinetics of enzyme reactions involving solid substrates (cellulase-cellulose), enzyme production and utilization, regulation of both primary and secondary metabolite production and the use, particularly for microbial protein production, of gaseous, liquid and solid sources of carbon. As might be expected, the approach of the individual contributors varies with the nature of the particular topic. A survey style has been adopted to the properties of fluids and enzymes; the chapter on metabolite production is descriptive, while consideration of alternative carbon sources is in the form of a review. The chapters on the recovery of microbial mass and enzyme kinetics are largely mechanistic in the biochemical engineering sense.

The authors are to be congratulated on their presentations and for collecting a substantial amount of information, both qualitative and quantitative, on the various topics. The result is an easily readable text which will broaden the outlook of the workers in the area.

\section{B. ATKINSON}

\section{Chemistry in the Cold}

Low Temperature Spectroscopy. By Beat Meyer. Pp. xi+653. (Elsevier: Amsterdam; American Elsevier: New York, 1971.) Dfl.138; \$38.50; $£ 15.70$.

WITH the invention of the vacuum flask by Dewar a whole new field of lowtemperature chemistry opened up. One important aspect of this has been the application of spectroscopic techniques, particularly when combined with the use of frozen solvents or matrices. Lewis and Porter saw the importance of photochemical processes of species in frozen organic glasses and Pimentel made a major advance by using frozen inert gases at $20 \mathrm{~K}$ as matrix materials. Such studies have concentrated on (a) primary photochemical processes-fluorescence, phosphorescence of isolated molecules including the perturbation of the guest energy levels by the host; $(b)$ the obtaining of spectra otherwise difficult to obtain, for example, Weltner's work on species of astrophysical interest; (c) the generation and examination of new unstable molecules, stabilized by a combination of low temperature and matrix isolation. The spectroscopic techniques include ultraviolet/visible, infrared (and very recently Raman) and electron spin resonance.

This book largely reflects the author's own interest in primary photochemistry. Indeed the preface states that "the book resulted from the author's need to have a text for undergraduates, $\mathrm{PhD}$ students and visiting scientists". Within these terms of reference the book succeeds admirably. For example, chapters 7 and 8 (111 pages altogether) provide a detailed description of apparatus and sample production; these chapters can be recommended to anyone contemplating entering the field. The earlier chapters in which are discussed the nature of spectra, photochemical processes and diffusion, however, are rather specialized. For example, chapter 4, "Details of Observed Spectra", is concerned exclusively with the effect of matrix on ultraviolet/visible spectra of isolated molecules-this ignores the considerable discussion in the literature of matrix effects on vibrational and electron spin resonance spectra. The book in fact contains very little about infrared and nothing about electron spin resonance spectra; this is a pity because such spectra have been of prime importance in establishing the generation of new species. There is also no mention of Rochkind's development of pseudo (or pulsed) matrix isolation, a technique of considerable analytical potential. Pages 233 to 493 provide a comprehensive summary of individual species up to July 1969 with some later references; this section, together with an extensive bibliography, is extremely valuable. Spectroscopic data recorded there are ultraviolet/visible and infrared which makes the absence of a discussion of infrared techniques more pointed.

Some specific points. The author is to be congratulated on providing so many illustrations, but just a few of them are rather misleading: for example, Fig. 3.2 does not match its caption; Fig. $8.6(a),(b)$ and $(c)$ do not match the text, and there is no Fig. $8(d)$ at all; the scale drawings of matrix materials in Fig. 6.4 do not agree with the lattice parameters in Table 8.2. There are few omissions and misprints - the reviewer only noticed the following: in the equation on page 25 the terms are not properly defined; the data for $\mathrm{SnS}$ in Table 3.2 cannot match the equation on page 27 ; on page $290 \mathrm{HNS}$ should be HNSi.

In short, this is a good book for a limited audience. J. J. TURNER 